\title{
THEOREM OF KURATOWSKI-SUSLIN FOR MEASURABLE MAPPINGS
}

\author{
ANDRZEJ WISNIEWSKI
}

(Communicated by Andrew M. Bruckner)

\begin{abstract}
The purpose of this paper is to describe these Borel mappings on a separable complete metric space $X$ which transform every measurable set (with respect to some measure $\mu$ on $X$ ) onto a measurable one. It is shown that a one-to-one Borel mapping $f$ on $X$ fulfills the above property if and only if the measure $\mu$ is absolutely continuous with respect to the measure $\mu_{f}$ (an image of $\mu$ under the mapping $f$ ). Our results are a generalization of the classical results of Suslin and Kuratowski.
\end{abstract}

\section{INTRODUCTION}

In his paper [4], Kuratowski proved the following theorem: Let $X$ be a separable complete metric space and $f$ a one-to-one Borel mapping on $X$. Then for every Borel subset $B$ of $X$ the image $f(B)$ is also a Borel set.

In the case of continuous mappings the above theorem has been already shown by Suslin in [9].

In the present paper we shall generalize the above theorem of Kuratowski, considering the measurability (of sets $B$ and $f(B)$ ) with respect to some measure on $X$ instead of their Borel measurability.

Denote by $\mathfrak{B}=\mathfrak{B}(X)$ the Borel $\sigma$-algebra on a separable complete metric space $X$ and let $\mu$ be a Borel measure on $X$. By $\mathfrak{B}_{\mu}$ we will denote the completion in measure $\mu$ of the $\sigma$-algebra $\mathfrak{B}$.

In this paper we shall consider therefore the question of characterizing of functions $f$ on $X$ such that $f(B) \in \mathfrak{B}_{\mu}$ if $B \in \mathfrak{B}_{\mu}$. It will be shown (Example 1) that the condition that $f$ is a one-to-one Borel mapping as in the theorem of Kuratowski is not enough. Using Lemma 1 we shall then prove that for $\mu$ nonatomic and $f$-onto mappings, the above problem is true iff $\mu \ll \mu_{f}$, i.e., the measure $\mu$ is absolute continuous with respect to the measure $\mu_{f}$, where $\mu_{f}$ denotes the image of measure $\mu$ under the mapping $f$.

Received by the editors May 20, 1992 and, in revised form, August 18, 1993.

1991 Mathematics Subject Classification. Primary 28A05, 28A20, 28C15; Secondary 28C20, 60B05, 60B11.

Key words and phrases. Borel sets, measurable and nonmeasurable sets, Borel mappings, measurable mappings, absolute continuity of measures, admissible translations of measures. 
Now we give an example which shows that the extension of the theorem of Kuratowski is impossible even in the case of measurability with respect to the Lebesgue measure on $R$.

Example 1. Let $m$ be the Lebesgue measure defined on the Borel $\sigma$-algebra $\mathfrak{B}$ of a real line $R$. Then $\mathfrak{B}_{m}$ is the $\sigma$-algebra of sets which are measurable with respect to $m$.

In the first place we show that there exists a one-to-one Borel mapping $f$ from $R$ into $R$ and there exist Borel sets $A$ and $B$ such that $m(B)=0, m(A)=1$, and $f(B)=A$.

Let $C$ be the Cantor set on $[0,1]$. It is well known that there exists a continuous mapping $g$ from $C$ onto $[0,1]$ and countable sets $B_{0} \subset C$ and $A_{0} \subset[0,1]$ such that $g$ is the one-to-one mapping from $C-B_{0}$ onto $[0,1]-A_{0}$ (see [3]). Therefore, if we put $B=C-B_{0}$ and $A=[0,1]-A_{0}$, then $B$ and $A$ are Borel sets such that $m(B)=0, m(A)=1$, and $g(B)=A$. If we now define a map $f: R \rightarrow R$ setting

$$
f(x)= \begin{cases}g(x) & \text { for } x \in B, \\ x+1 & \text { for } x \in(R-B) \cap[0,+\infty), \\ x & \text { for } x \in(-\infty, 0),\end{cases}
$$

then $f$ is a one-to-one Borel mapping from $R$ onto $R$ such that $f(B)=A$.

Now we show that for this function $f$ there exists a set $E$ which belongs to the $\sigma$-algebra $\mathfrak{B}_{m}$ and such that $f(E) \notin \mathfrak{B}_{m}$.

Indeed, since $A \in \mathfrak{B}$ and $m(A)=1$, there is a set $D \subset A$ such that $D \notin \mathfrak{B}_{m}$. Let $E=f^{-1}(D)$. Since $D \subset A$, it follows that $g^{-1}(D) \subset g^{-1}(A)$. But $A=g(B)$ and $g$ is an injection that implies $g^{-1}(A)=g^{-1}(g(B))=B$. Thus $E \subset B$. However $m(B)=0$, whence we conclude that $E \in \mathfrak{B}_{m}$. But $f(E)=f\left(f^{-1}(D)\right)=D$. Therefore $f(E) \notin \mathfrak{B}_{m}$ completes Example 1 .

In the above example we used a well-known fact that if $A$ is a Borel subset of $R$ with positive Lebesgue measure, then there exists a subset $B$ of $A$ which is nonmeasurable with respect to $m$ (see, e.g., [5]). In this paper we will need some extension of this fact.

Lemma 1. Let $X$ be a separable complete metric space and $\mathfrak{B}=\mathfrak{B}(X)$ the Borel $\sigma$-algebra on $X$. Suppose that $\mu$ is a $\sigma$-finite and nonatomic measure on $\mathfrak{B}$. If $A \in \mathfrak{B}$ and $\mu(A)>0$, then there exists a set $B \subset A$ such that $B \notin \mathfrak{B}_{\mu}$.

Proof. It is well known that by our assumptions the measure space $(X, \mathfrak{B}, \mu)$ is isomorphic (in the sense of measure theory) to the space $(R, \mathfrak{B}(R), m)$, where $m$ is the Lebesgue measure on $R$ [6, Theorem 26.6]. This means that there exist Borel sets $X_{0} \subset X$ and $Y_{0} \subset R$ such that $\mu\left(X_{0}\right)=0, m\left(Y_{0}\right)=0$ and there is a one-to-one map $T$ from $X-X_{0}$ onto $R-Y_{0}$ such that $T$ and $T^{-1}$ are Borel mappings and $m$ is an image of $\mu$ under $T$, i.e., for every Borel set $E \subset R-Y_{0}$ we have

$$
\mu\left(T^{-1}(E)\right)=m(E) .
$$

Let $A_{1}=A \cap\left(X-X_{0}\right)$. Then $A_{1} \in \mathfrak{B}$ and $\mu\left(A_{1}\right)>0$. Since $T^{-1}$ is a Borel map, it follows that $T\left(A_{1}\right) \in \mathfrak{B}(R)$ and moreover from (1) we have that 
$m\left(T\left(A_{1}\right)\right)=\mu\left(A_{1}\right)$. Thus $m\left(T\left(A_{1}\right)\right)>0$. Hence there is a set $C \subset T\left(A_{1}\right)$ such that $C \notin \mathfrak{B}_{m}(R)$. Let $B=T^{-1}(C)$. Of course, $B \subset A_{1}$ and consequently $B \subset A$.

We shall prove that $B$ is nonmeasurable with respect to $\mu$, i.e., $B \notin \mathfrak{B}_{\mu}$. Contrary to this, let us suppose that $B \in \mathfrak{B}_{\mu}$. Then $B=D_{1} \cup D_{2}$ where $D_{1} \in \mathfrak{B}$ and $D_{2} \subset N$ for some $N \in \mathfrak{B}$ with $\mu(N)=0$. Moreover, we may obviously assume that $N \subset X-X_{0}$. Therefore $T^{-1}(C)=D_{1} \cup D_{2}$ and consequently $T\left(T^{-1}(C)\right)=T\left(D_{1} \cup D_{2}\right)$, i.e., $C=T\left(D_{1}\right) \cup T\left(D_{2}\right)$. But $D_{1} \in \mathfrak{B}$ and $N \in \mathfrak{B}$ whence $T\left(D_{1}\right) \in \mathfrak{B}(R)$ and $T(N) \in \mathfrak{B}(R)$. Moreover, from (1) we have that $m(T(N))=\mu(N)=0$. Hence, since $T\left(D_{2}\right) \subset T(N)$, we obtain that $T\left(D_{2}\right) \in \mathfrak{B}_{m}(R)$, and consequently also $C \in \mathfrak{B}_{m}(R)$, which is a contradiction and completes our proof.

\section{MAIN Results}

In view of Example 1 we see that the generalization of the theorem of $\mathrm{Ku}$ ratowski to the case of measurability with respect to some measure need not always be true.

The main result of this paper gives the conditions under what such generalization is possible.

Theorem 1. Let $X$ be a separable complete metric space, $\mathfrak{B}$ the Borel $\sigma$-algebra on $X$, and $\mu$ a measure on $\mathfrak{B}$. Suppose that $f$ is a one-to-one Borel mapping from $X$ into $X$. In order to $f(B) \in \mathfrak{B}_{\mu}$ for every $B \in \mathfrak{B}_{\mu}$ it is sufficient, and if $\mu$ is nonatomic and $\sigma$-finite and $f$ is a surjection, it is also necessary that $\mu \ll \mu_{f}$.

Proof. Sufficiency. Suppose that $\mu \ll \mu_{f}$ and let $B \in \mathfrak{B}_{\mu}$, i.e., $B=A \cup N$ where $A \in \mathfrak{B}, N \subset A_{1}, A_{1} \in \mathfrak{B}, \mu\left(A_{1}\right)=0$. We have that

$$
f(B)=f(A) \cup f(N) .
$$

Since $A \in \mathfrak{B}$, it follows that $f(A) \in \mathfrak{B}$ and consequently $f(A) \in \mathfrak{B}_{\mu}$.

Now we show that also $f(N) \in \mathfrak{B}_{\mu}$. Indeed, from $A_{1} \in \mathfrak{B}$ it follows that $f\left(A_{1}\right) \in \mathfrak{B}$. Moreover, since $f$ is an injection, we have that $f^{-1}\left(f\left(A_{1}\right)\right)=A_{1}$, whence $\mu_{f}\left(f\left(A_{1}\right)\right)=\mu\left(f^{-1}\left(f\left(A_{1}\right)\right)\right)=\mu\left(A_{1}\right)$. But $\mu\left(A_{1}\right)=0$. Therefore $\mu_{f}\left(f\left(A_{1}\right)\right)=0$. Thus in view of the assumption (i.e., $\mu \ll \mu_{f}$ ) we receive that $\mu\left(f\left(A_{1}\right)\right)=0$. But $N \subset A_{1}$ implies that $f(N) \subset f\left(A_{1}\right)$. Hence $f(N) \in \mathfrak{B}_{\mu}$.

We proved therefore that $f(A) \in \mathfrak{B}_{\mu}$ and $f(N) \in \mathfrak{B}_{\mu}$, which by virtue of (2) implies that $f(B) \in \mathfrak{B}_{\mu}$ and ends the proof of sufficiency.

Necessity. Suppose that our assertion is not true. This means that there exists a set $B_{1} \in \mathfrak{B}$ such that $\mu_{f}\left(B_{1}\right)=0$ and $\mu\left(B_{1}\right)>0$. From Lemma 1 we receive that there is a set $A_{1} \subset B_{1}$ such that $A_{1} \notin \mathfrak{B}_{\mu}$. Let $B=f^{-1}\left(A_{1}\right)$. Since $A_{1} \subset B_{1}$, it follows that $f^{-1}\left(A_{1}\right) \subset f^{-1}\left(B_{1}\right)$, i.e., $B \subset f^{-1}\left(B_{1}\right)$. But $f^{-1}\left(B_{1}\right) \in \mathfrak{B}$ and $\mu\left(f^{-1}\left(B_{1}\right)\right)=\mu_{f}\left(B_{1}\right)=0$, which implies that $B \in \mathfrak{B}_{\mu}$. On the other hand, we have, however, that $f(B)=f\left(f^{-1}\left(A_{1}\right)\right)=A_{1}$ (since $f(X)=X)$. But $A_{1} \notin \mathfrak{B}_{\mu}$, whence $f(B) \notin \mathfrak{B}_{\mu}$. Therefore we have found a set $B \in \mathfrak{B}_{\mu}$ such that $f(B) \notin \mathfrak{B}_{\mu}$, which contradicts the assumption and consequently completes the proof of necessity in Theorem 1 .

As an illustration of our results we consider now the case of translations in a linear space. It appears also that for such simple mappings, the generalization of the theorem of Kuratowski that we treat need not be true (Example 2). Then 
from Theorem 1 we shall receive a characterization of these translations on the linear metric space $X$ with measure $\mu$ for which such a generalization is true (Theorem 2).

Thus let $X$ be a real separable complete linear metric space. For $x \in X$ denote by $T_{x}$ the translation by $x$, i.e., $T_{x}(y)=y+x$ for $y \in X$. Of course, for every $x \in X \quad T_{x}$ is a one-to-one Borel mapping on $X$. Therefore for each such translation the theorem of Kuratowski holds true, which means that the Borel $\sigma$-algebra $\mathfrak{B}=\mathfrak{B}(X)$ is invariant under all translations, i.e., $B+x \in \mathfrak{B}$ for any $x \in X$ and $B \in \mathfrak{B}$.

Now let $\mu$ be a Borel measure on $X$. In some cases the generalization of the theorem of Kuratowski is then true, which also means that the $\sigma$-algebra $\mathfrak{B}_{\mu}$ is invariant under all translations; for example, if $X=R^{n}$ and $\mu$ is the Lebesgue measure on $R^{n}$ (see, e.g., [6]). But the situation may change if $X$ is an infinitely-dimensional linear space.

Example 2. Let $\nu$ be a Gaussian probability measure on $R$, and let $\mu=\nu \times \nu \times$ .. be the product measure defined on the linear metric space $R^{\infty}=R \times R \times \cdots$. We will show that the $\mu$-completion of the Borel $\sigma$-algebra $\mathfrak{B}=\mathfrak{B}\left(R^{\infty}\right)$ is not invariant under translations.

Let us remark that there exists a set $Y \subset R^{\infty}$ which is not $\mu$-measurable, i.e., $Y \notin \mathfrak{B}_{\mu}[7$, p. 92].

Now let $A=\left\{x=\left(x_{n}\right) \in R^{\infty}: \sum x_{n}^{2} / n^{2}<\infty\right\}$. Then $A$ is a Borel subset of $R^{\infty}$ and $\mu(A)=1$ (see [7, p. 54]). It is easy to see that if we define an element $y=\left(y_{n}\right) \in R^{\infty}$ putting $y_{n}=n(n=1,2, \ldots)$, then $A \cap(A+y)=\varnothing$. Therefore, since $\mu(A)=1$ and $\mu$ is a probability measure, the set $A+y$ is $\mu$-measurable and $\mu(A+y)=0$. Now we put $C=A \cap Y$. Let us remark that $C \notin \mathfrak{B}_{\mu}$. Indeed, since $\mu(A)=1$, it follows that $Y-A \in \mathfrak{B}_{\mu}$. If we now suppose that $C \in \mathfrak{B}_{\mu}$, then from $Y=C \cup(Y-A)$ we obtain that $Y \in \mathfrak{B}_{\mu}$. But $Y$ is not a $\mu$-measurable set. Therefore $C \notin \mathfrak{B}_{\mu}$.

Now we define a set $B$ putting $B=C+y$. Since $C \subset A$, it follows that $C+y \subset A+y$, i.e., $B \subset A+y$. But $A+y \in \mathfrak{B}_{\mu}$ and $\mu(A+y)=0$. Hence $B \in \mathfrak{B}_{\mu}$. But on the other hand $B-y=C$ and $C \notin \mathfrak{B}_{\mu}$. Therefore $B-y \notin \mathfrak{B}_{\mu}$. Thus the $\sigma$-algebra $\mathfrak{B}_{\mu}$ of $\mu$-measurable sets is not invariant under translations.

If $\mu$ is a measure on a linear space $X$ and $T_{x}(x \in X)$ is a translation on $X$, we shall denote for simplicity the measure $\mu_{T_{x}}$ by $\mu_{x}$. An element $x \in X$ for which $\mu \ll \mu_{x}$ is called an admissible translation of the measure $\mu$. Denote by $A S(\mu)$ the set of all admissible translations of $\mu$, i.e., $A S(\mu)=\{x \in X$ : $\mu \ll \mu_{X}$ \}. From Theorem 1 we thus obtain the following theorem:

Theorem 2. Let $\mu$ be a $\sigma$-finite and nonatomic measure defined on the Borel $\sigma$-algebra $\mathfrak{B}$ of a separable complete linear metric space $X$, and let $x \in X$. $B+x \in \mathfrak{B}_{\mu}$ for every set $B \in \mathfrak{B}_{\mu}$ if and only if $x \in A S(\mu)$.

The set of admissible translations for various measures $\mu$ has been studied by a lot of authors. The detailed structure of $A S(\mu)$ for specific $\mu$ was given in $[1,2,8,10]$.

At the end of this paper we give some remarks about the possibility of further generalization of the theorem of Kuratowski that we considered. Namely, it is easy to prove that Theorem 1 holds true also for $\mu$-measurable mappings on a 
separable complete metric space $X$, i.e., measurable with respect to $\left(\mathfrak{B}_{\mu}, \mathfrak{B}\right)$. Moreover, in the similar way as in Theorem 1 , it is simple to show that if $\mu$ is $\sigma$-finite and nonatomic, then for every $\mu$-measurable mapping $f$ from $X$ into $X f^{-1}(B) \in \mathfrak{B}_{\mu}$ for every $B \in \mathfrak{B}_{\mu}$ iff $\mu_{f} \ll \mu$.

\section{REFERENCES}

1. S. D. Chatterji, Singularity and absolute continuity of measures in infinite dimensional spaces, Lecture Notes in Math., vol. 656, Springer-Verlag, Berlin, Heidelberg, and New York, 1978, pp. 17-23.

2. S. D. Chatterji and V. Mandrekar, Quasi-invariance of measures under translation, Math. Z. 154 (1977), 19-29.

3. R. Engelking, General topology, PWN, Warszawa, 1977.

4. K. Kuratowski, Topology I, Academic Press, New York, and PWN, Warszawa, 1966.

5. I. P. Natanson, Theorie der Funktionen einer reellen Veranderlichen, Akademie-Verlag, Berlin, 1954.

6. K. R. Parthasarathy, Introduction to probability and measure, Mc-Millan Co. of India, Delhi, 1980.

7. G. E. Silov and Fan Dyk Tin, Integral, measure and derivative in linear spaces, Nauka, Moscow, 1967.

8. A. V. Skorohod, Integration in Hilbert spaces, Springer-Verlag, Berlin, Heidelberg, and New York, 1974.

9. M. Suslin, Sur une definition des ensembles mesurables $B$ sans nombres transfinis, C. R. Acad. Sci. Paris 164 (1917), 89.

10. J. Zinn, Admissible translates of stable measures, Studia Math. 54 (1976), 245-257. Poland

INSTitute OF MATHematics, SZCZecin University, Ul. WielKopolsKa 15, 70-451 SzCZecin,

E-mail address: awisniew@uoo.univ.szczecin.pl 